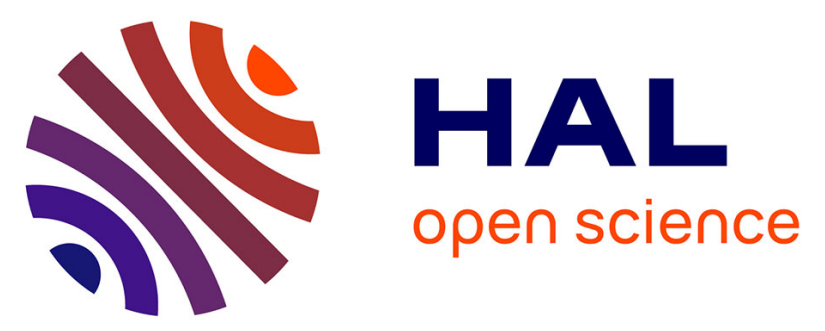

\title{
Scanning the surface of soft tissues with a micrometer precision thanks to endomicroscopy based visual servoing
}

Benoît Rosa, Mustapha Suphi Erden, Tom Vercauteren, Jérome Szewczyk, Guillaume Morel

\section{To cite this version:}

Benoît Rosa, Mustapha Suphi Erden, Tom Vercauteren, Jérome Szewczyk, Guillaume Morel. Scanning the surface of soft tissues with a micrometer precision thanks to endomicroscopy based visual servoing. 2012 IEEE/RSJ International Conference on Intelligent Robots and Systems (IROS 2012), Oct 2012, Vilamoura-Algarve, Portugal. 10.1109/IROS.2012.6385643 . hal-01765059

\section{HAL Id: hal-01765059 \\ https://hal.science/hal-01765059}

Submitted on 12 Apr 2018

HAL is a multi-disciplinary open access archive for the deposit and dissemination of scientific research documents, whether they are published or not. The documents may come from teaching and research institutions in France or abroad, or from public or private research centers.
L'archive ouverte pluridisciplinaire HAL, est destinée au dépôt et à la diffusion de documents scientifiques de niveau recherche, publiés ou non, émanant des établissements d'enseignement et de recherche français ou étrangers, des laboratoires publics ou privés. 


\title{
Scanning the surface of soft tissues with a micrometer precision thanks to endomicroscopy based visual servoing
}

\author{
Benoît Rosa ${ }^{1}$, Mustapha Suphi Erden ${ }^{1}$, Tom Vercauteren ${ }^{2}$, Jérôme Szewczyk ${ }^{1}$, and Guillaume Morel ${ }^{1}$
}

\begin{abstract}
Probe-based confocal laser endomicroscopy is a recent tissue imaging technology that requires placing a probe in contact with the tissue to be imaged and provides real time images with a microscopic resolution. Additionally, generating adequate probe movements to sweep the tissue surface can be used to reconstruct a wide mosaic of the scanned region while increasing the resolution which is appropriate for anatomicopathological cancer diagnosis.

However, properly controlling the motion along the scanning trajectory is a major problem. Indeed, the tissue exhibits deformations under friction forces exerted by the probe leading to deformed mosaics. In this paper we propose a visual servoing approach for controlling the probe movements relative to the tissue while rejecting the tissue deformation disturbance. The probe displacement with respect to the tissue is firstly estimated using the confocal images and an image registration real-time algorithm. Secondly, from this real-time image-based position measurement, the probe motion is controlled thanks to a simple proportional-integral compensator and a feedforward term. $E x$ vivo experiments using a Stäubli TX40 robot and a Mauna Kea Technologies Cellvizio imaging device demonstrate the effectiveness of the approach on liver and muscle tissue. Index Terms-Medical robotics, medical imaging, image registration, probe-based confocal laser endomicroscopy, soft tissue deformation, visual servoing
\end{abstract}

\section{INTRODUCTION}

Biopsy is one of the key elements in cancer treatment procedure. It aims at analyzing a piece of tissue under a microscope in order to determine whether it contains cancer cells. The piece of tissue is extracted by the surgeon and brought to the analysis lab where it is frozen, cut into pieces and analyzed under a microscope. This procedure is time consuming and invasive. Alternatively, recently developed imaging techniques, such as optical coherence tomography [1] and confocal endomicroscopy [2], [3], have the potential to provide an in vivo imaging resolution similar to the optical microscopes used for biopsy analysis, in realtime and without tissue damage.

Several studies reported successful examinations ex vivo [4], and in vivo in the gastrointestinal tract [5], [6] and in the abdominal cavity [7]. One common problem is that the image field of view (typically a few hundred micrometers) is not always wide enough to allow a conclusive diagnosis based on the confocal images. Therefore, mosaicing algorithms have been developed [8], [9]. Smoothly and precisely

\footnotetext{
${ }^{1}$ UPMC Univ Paris 06, UMR 7222, ISIR, F-75005, Paris, France and CNRS, UMR 7222, ISIR, F-75005, Paris, France (rosa, erden, szewczyk, morel) at isir.upmc.fr

${ }^{2}$ Mauna Kea Technologies, F-75010, Paris, France tom at maunakeatech.com
}

sweeping the probe along the tissue surface allows to collect images that can be blended in a single mosaic with a wider field of view. However, the precision and stability required for such a motion makes it difficult to obtain good quality mosaics using a manual actuation of the probe. Therefore, robotized means have been proposed for the stabilization of the probe on the tissue and its actuation [10], [11].

A major problem for tissue scanning is the local deformation of the tissue caused by the adherence with the imaging probe. Namely, the motion imposed to the probe, relative to a fixed frame, does not match the motion of the probe relative to the scanned tissue area, which, to a certain extend, sticks to the probe. Attempts to model tissue deformations under contact forces can be found in the literature [12]-[14]. However, these models involve parameters that strongly vary with local conditions (humidity, tissue stiffness, etc.). In that sense, their use for predicting the deformation is difficult to implement.

In this article, we propose to use vision based control of the probe motion in order to reject the tissue deformation disturbance. We propose a method for simultaneously measuring the probe motion with respect to a rigid frame and relative to the tissue. Using this data, we highlight and explain the tissue deformation problem and propose a control scheme for compensating it, based on the visual servoing approach.

\section{EXPERIMENTAL SETUP}

The experimental platform is presented on Fig. 1. It consists of a confocal endomicroscope with its probe, a Stäubli TX40 6 DoF robot for generating the probe movements, and a tissue to be imaged that is placed under the probe on a rigid surface.

The confocal endomicroscope is a Cellvizio device designed and commercialized by Mauna Kea Technologies, Paris, France. The Gastroflex UHD probe has the shape of a flexible fiber bundle with an optical head at its tip. The fiber bundle has a diameter of $1.4 \mathrm{~mm}$ and the optical head has an outer diameter of $2.6 \mathrm{~mm}$. The excitation wavelength is $488 \mathrm{~nm}$, and the contrast agent for fluorescence marking of the tissues is, for our ex vivo setup, Acriflavin. The images are acquired at 12 frames/s. They present a $1 \mu \mathrm{m}$ lateral resolution and a field of view of $240 \mu \mathrm{m} \times 200 \mu \mathrm{m}$. The depth of the focal plan is $50 \mu \mathrm{m}$ under the tissue surface.

During the experiments the probe was fixed at the end effector of the robot thanks to a rapidly prototyped probe holder. The robot has 6 degrees of freedom and a 20 microns repeatability at its end effector. The surface where the tissue 


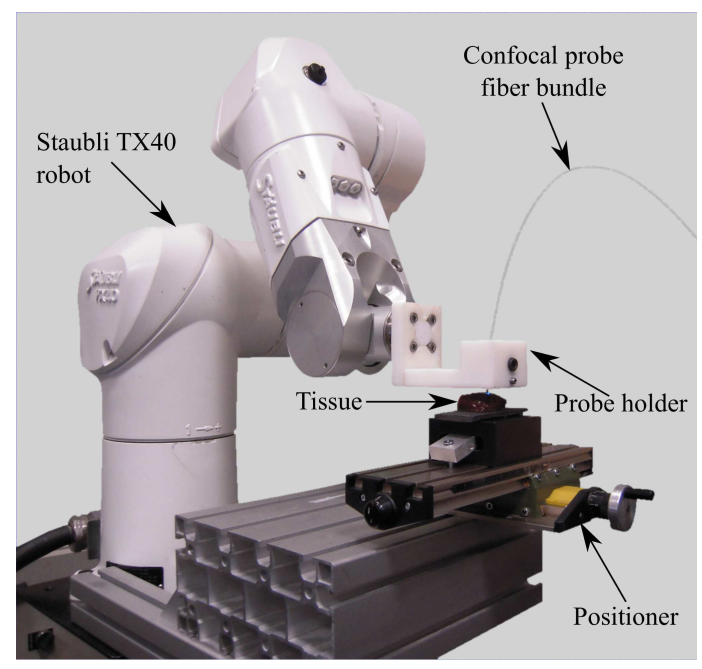

Fig. 1. Test bench using a Stäubli TX40 robot and the Cellvizio probe

samples were put was tightened to the robot base and set parallel to the XY horizontal plane using a digital inclinometer. Considering the small size of the scanned areas (a few square millimeters), the scanned tissues were considered as planar in this study. Therefore, the robot movements were generated at a constant height $\mathrm{Z}$. Meanwhile, the probe orientation is kept constant, which finally results in a 2 degrees of freedom planar positioning problem. The Cartesian position of the optical head is controlled by the onboard controller of the Staubli robot which is properly interfaced for allowing real time update.

\section{MEASURING THE PROBE-TISSUE RELATIVE MOTION}

This section focuses on the estimation of the local probe motion relative to the tissue. In the next, $V_{r / 0}, V_{p / 0}$ and $V_{a / 0}$ denote the (planar translational) velocities of the robot (subscript $r$ ), the probe (subscript ${ }_{p}$ ) and the anatomical tissue (subscript ${ }_{a}$ ) relative to a fixed base (subscript ${ }_{0}$ ), respectively; $V_{p / a}$ and $V_{p / r}$ denote the velocities of the probe relative to the tissue and the robot, respectively. Composition of the planar translational movements leads to:

$$
\begin{aligned}
& V_{p / a}=V_{p / 0}-V_{a / 0} \\
& V_{p / 0}=V_{p / r}+V_{r / 0}
\end{aligned}
$$

Equation (1) highlights the fact that the probe motion relative to the tissue, which corresponds to the movement in the image used for producing mosaics, depends on the tissue deformations. Equation (2) highlights the fact that the robot speed relative to the fixed frame may differ from the probe speed relative to the fixed frame due to possible deformations of the probe holder. Recall that tiny deformations of a few micrometers have to be considered here due to the image sensor resolution. Therefore, $V_{p / r}$ cannot be a priori neglected.

\section{A. Image-based relative motion estimation}

For proper control of the probe motion along the tissue surface, an estimation of $V_{p / a}$ is required. To this aim, we rely on registration between two successive images and make the assumption that the motion of the probe can be modeled by a constant translation velocity on any time frame corresponding to the acquisition of a pair of successive images. This assumption allows us to perform image registration in real-time as presented in [15] with the drawback that only probe-tissue translations, with potentially time-varying velocity, can be recovered. This limitation is not restrictive with the current setup. Indeed, the robotic control imposes a translation of the probe and there is no physical reason for generating significant torsional deformation of the tissue under these conditions. Therefore, it is assumed that the rotation of the probe with respect to the tissue is small enough to be neglected.

Most visual tracking methods rely on an initialization and an optimization procedure. Most methods are therefore subject to local minima. On the contrary, although being restricted to finding translations, the fast normalized correlation method of [16] provides a global optimum, which is highly needed when dealing with images showing little contrast or texture as those observed with our system. Given two consecutive confocal images $I_{a}$ and $I_{b}$, the main idea is to evaluate, in one pass with a Fourier transform, the correlation coefficient for every possible translation $\zeta$ having integer components:

$\operatorname{Sim}\left(I_{a}, I_{b} \circ \zeta\right)=\frac{\sum_{k}\left(I_{a}(k)-\bar{I}_{a}\right)\left(I_{b}(k+\zeta)-\bar{I}_{b}\right)}{\sqrt{\sum_{k}\left(I_{a}(k)-\bar{I}_{a}\right)^{2} \sum_{k}\left(I_{b}(k+\zeta)-\bar{I}_{b}\right)^{2}}}$

where $\bar{I}_{x}$ is the mean of $I_{x}$ and $k$ stands for a pixel index. Given the full correlation coefficient map, we simply extract its maximum to get the optimal translation $\hat{\zeta}\left(I_{a}, I_{b}\right)$. Fast normalized correlation matching provides globally optimal results, can be computed efficiently and requires a fixed computational budget. This makes it an ideal candidate for real-time applications. Nonetheless, it has been designed for template matching rather than image matching and is theoretically correct only if the support of $I_{b} \circ \zeta$ is included in the support of $I_{a}$, which is almost never fulfilled when working with images having the same size. In practice, this non-optimality leads to border effects and a lack of symmetry in the results, i.e. $\hat{\zeta}\left(I_{a}, I_{b}\right) \neq-\hat{\zeta}\left(I_{b}, I_{a}\right)$. To alleviate the lack of symmetry of the algorithm, we rely on an average of the forward and backward estimation:

$$
\hat{\zeta}^{s}\left(I_{a}, I_{b}\right)=0.5\left(\hat{\zeta}\left(I_{a}, I_{b}\right)-\hat{\zeta}\left(I_{b}, I_{a}\right)\right)
$$

As demonstrated in [15] this approach provides reliable registration results.

Given the estimation $\hat{\zeta}^{s}$ of the translation in pixels between two consecutive images, we now need to estimate the translation in microns on the tissue. Factory calibration of the probe provides the scaling factor $s p$ from which a first 

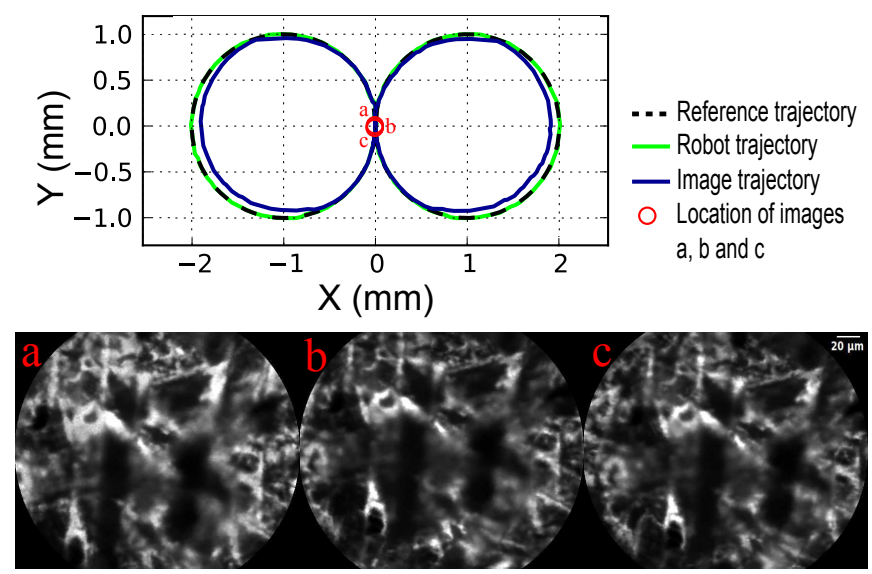

Fig. 2. 8-shape trajectory on a sheet of paper. Up: XY graph for comparison of robot and image trajectory. Down: first (a), middle (b) and last (c) confocal images

estimate of the velocity writes:

$$
\widehat{V}_{p / a}^{u}=\frac{s p}{T_{a c q}} \hat{\zeta}^{s}
$$

where $T_{a c q}$ is the acquisition time of an image. However, as explained in [8], the imager is a scanning device and compensating for the distortions of an image due to the motion is required. Following the derivations in [8] the estimated distortion-compensated velocity $\widehat{V}_{p / a}$ is:

$$
\widehat{V}_{p / a}=\frac{1}{1-\frac{\left(\widehat{V}_{p / a}^{u}\right)_{y}}{V_{y}^{\text {mirror }}}} \widehat{V}_{p / a}^{u}=\frac{1}{1-\frac{\left(\widehat{V}_{p / a}^{u}\right)_{y}}{V_{y}^{\text {mirror }}}} \frac{s p}{T_{a c q}} \hat{\zeta}^{s}
$$

where $V_{y}^{\text {mirror }}$ is the vertical speed of the scanning and $\left(\widehat{V}_{p / a}^{u}\right)_{y}$ is the projection of $\widehat{V}_{p / a}^{u}$ on the $y$ axis of the image frame.

The Cellvizio controller computes $\widehat{V}_{p / a}$ in real-time and sends the result via an ethernet link to the robot controller. The integration of this data over time gives the current image position relative to the beginning of the trajectory:

$$
\widehat{X}_{p / a}(t)=\widehat{X}_{p / a}(0)+\int_{0}^{t} \widehat{V}_{p / a}(\tau) d \tau
$$

In the next, the trajectory of $\widehat{X}_{p / a}(t)$ is called the image trajectory.

Finally, note that the extrinsic parameters of the imaging device, i.e. the constant orientation of the probe frame relative to the robot end effector frame, need to be identified. It is achieved by generating straight line motions of the probe along known directions in the robot frame and measuring the direction of the resulting image trajectory.

\section{B. Validation}

Since the image trajectory computation relies on the timeintegration of a speed measurement, it is subject to drift. For quantifying this effect, scans are firstly performed when the probe contacts a rigid surface, namely a sheet of paper. It is assumed that there is no local deformation at the paper surface in contact with the probe.
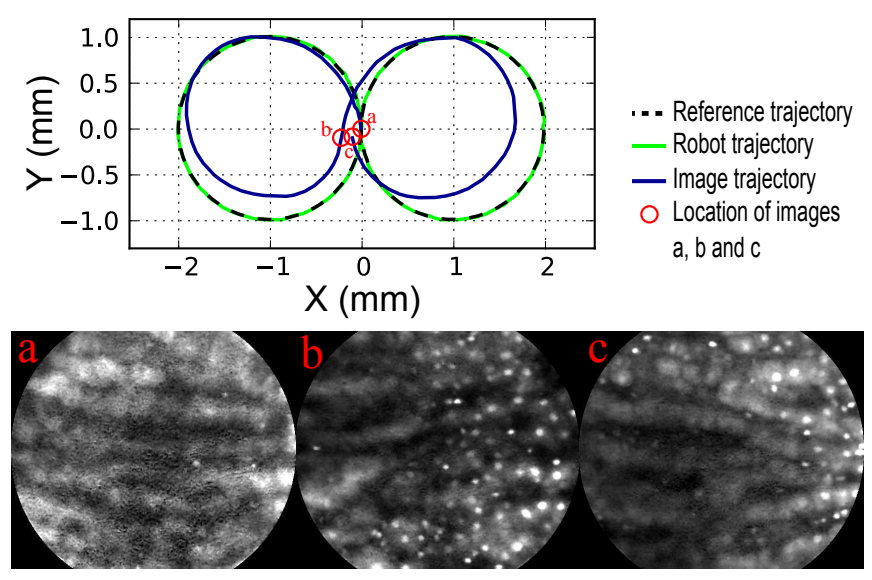

Fig. 3. 8-shape trajectory on a piece of beef liver. Up: XY graph for comparison of robot and image trajectory. Down: first (a), middle (b) and last (c) images

Figure 2 shows the results for an 8 -shape trajectory formed of two circles of $2 \mathrm{~mm}$ diameter each. This displacement is rather large as compared to the image resolution $(1 \mu \mathrm{m})$. It can be seen that the robot follows the desired trajectory with a high precision (desired speed is $0.3 \mathrm{~mm} / \mathrm{s}$ ) according to its own system of measurement. Moreover, the trajectory measured by image processing only sightly differs from the robot measured trajectory. The maximal error between the robot and image trajectorues is $140 \mu \mathrm{m}$. This can be explained by robot modeling errors and small deformations in the robot structure, the probe holder or the paper sheet support.

Most importantly, the first image (a), the last image (c), and the image in the middle of the trajectory (b) are very close to each other (see Fig. 2). Comparison of the displacement between images (a) and (c) for figure 2 and the same displacement measured using $\widehat{X}_{p / a}(t)$ gives an error of $6 \mu \mathrm{m}$. Considering the fact that this has been verified in repeated experiments, one can conclude that the drift of the image trajectory due to cumulative errors is negligible for a trajectory of a few millimetres long.

\section{SOFT TISSUE DEFORMATION}

Following the same procedure as in Section III-B, we generate an 8-shape trajectory on a soft tissue. A piece of beef liver is cut, marked with Acriflavin, and placed under the probe on a rough surface that avoids slipping of the tissue (see Fig. 1). The robot is then positionned with successive $100 \mu \mathrm{m}$ steps down until an image appears on the Cellvizio screen (the Cellvizio confocal probe provides images only when in contact). Then, a $300 \mu \mathrm{m}$ step down is made in order to guarantee proper contact conditions during the whole scan despite eventual lack of planarity. Finally, the 8-shape scan is performed. In order to avoid tissue drying, a drop of saline solution is regularly delivered on its surface.

The corresponding results are given in Fig. 3. One clearly sees that the image trajectory is shifted relatively to the robot trajectory. The confocal images (a), (b) and (c) do not overlap 
anymore, whereas the robot performed the same trajectory as for the sheet of paper experiment.

These results, compared to the rigid environment results (see Fig. 2), show that the tissue deformation is not negligible at this scale and has a direct impact on the trajectory effectively followed by the probe relatively to the tissue. In fact, similarly to what is described in [17], [18], when the probe starts moving at the contact of the tissue surface it first stretches the tissue because of friction forces. During this loading phase there is no motion of the probe relative to the tissue layer that is imaged and lies $50 \mu \mathrm{m}$ under the contact surface. Consequently, the robot moves a given distance while the probe does not move relatively to the tissue. After the loading phase, the probe enters in a slipping phase, when the drag force exerted on the tissue equals the friction force (see Fig. 4).
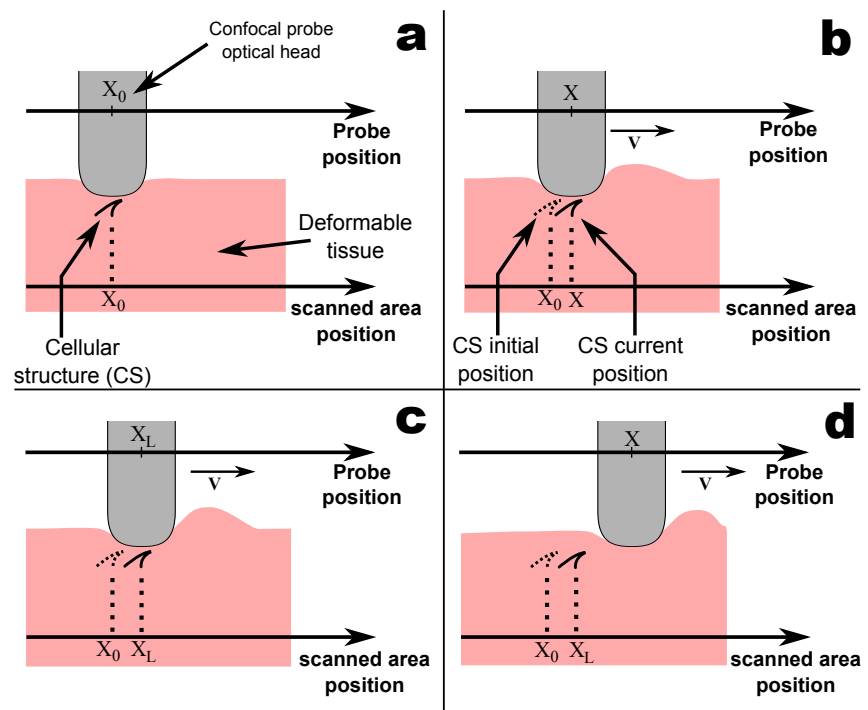

Fig. 4. Tissue behavior during probe motion: displacement of a cellular structure initially within the probe field of view. a: probe contact without motion. b: motion begins - loading phase. c: full load. d: slipping phase

In order to further illustrate the loading/slipping behavior, two additional experiments are performed on beef liver. In the first experiment, (Fig. 5), a forward-backwards $1 \mathrm{~mm}$ line scan in the $\mathrm{x}$ direction at $0.3 \mathrm{~mm} / \mathrm{s}$ is performed on rigid paper (Fig. 5a) and soft liver tissue (Fig. 5b).

For the soft tissue experiment, during the loading phases the image speed increases slowly when the robot moves. During the slipping phases, the robot and image speed are almost equal. This effect is emphasized when the robot goes back and forth because the tissue must first be unloaded before being loaded in the other direction, leading to a hysteretic behavior.

This is confirmed by an additional experiment, in which the commanded trajectory is a raster scan with soft tissue (Fig. 6). Though a visual inspection confirms the good quality of the mosaic, it presents holes and has a shape which is very far from the expected rectangle. This phenomenon results from a complex 2D coupling of the tissue deformations a

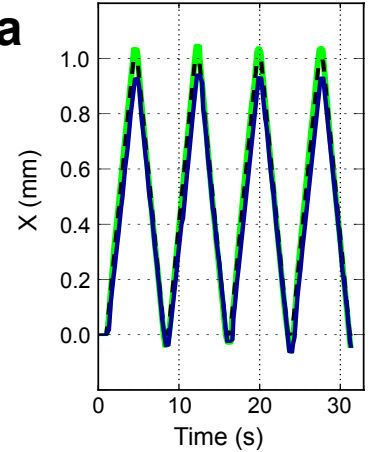

Desired
Robot

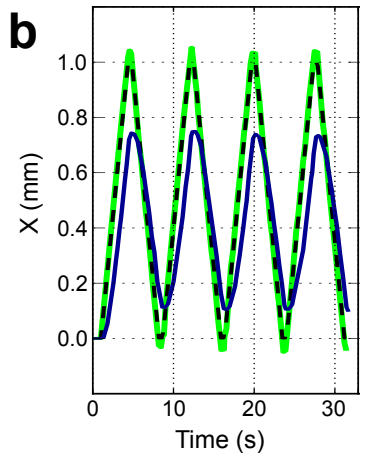

- Image
Fig. 5. Comparison of single line scan on paper (a) and liver (b)

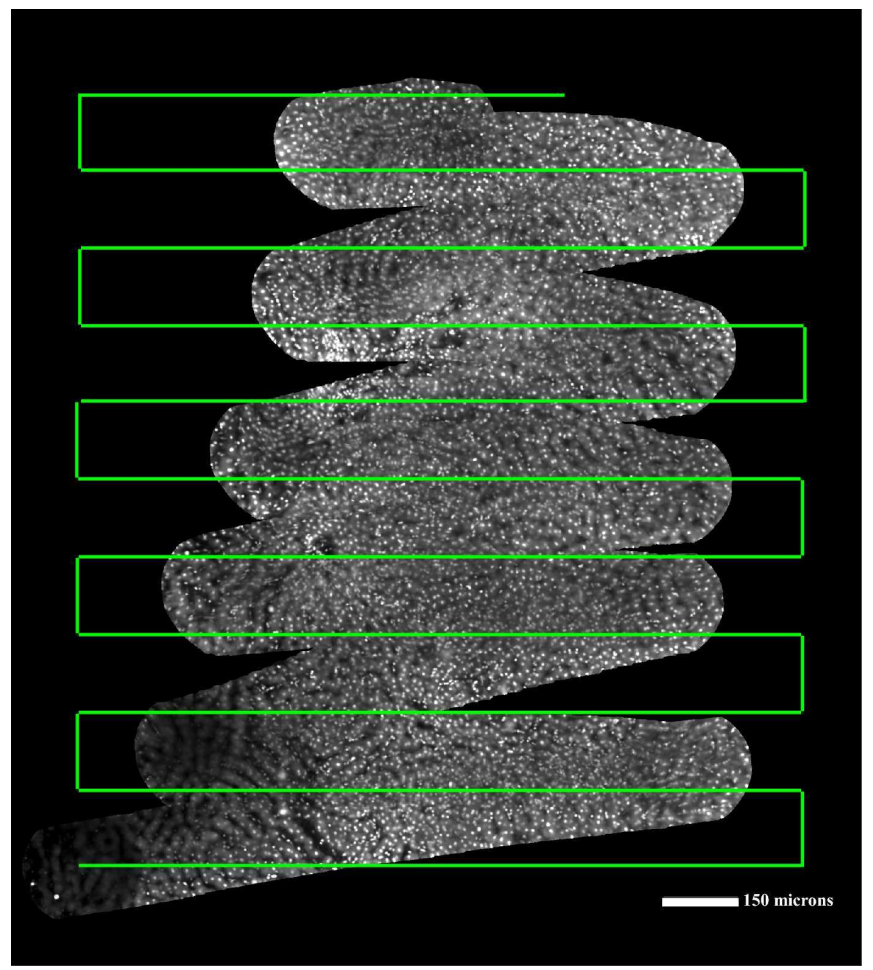

Fig. 6. Mosaic obtained following a raster scan on the surface of a piece of liver. The green line is the commanded trajectory of the confocal optical head center

under probe loading with changing directions and is hard to predict.

\section{Closing THE LOOP: VISUAL SERVOING}

It has been shown in Sec. IV that precisely controlling the probe motion with respect to the robot frame is not enough for obtaining a good control of the confocal probe motion with respect to the tissue surface.

In this section, we develop a model-free visual servoing approach exploiting the image-based measurement algorithm described by Eqs. (3-7). 


\section{A. Position control scheme}

The controller is aimed at computing the robot control input, which is the velocity $V_{r / 0}$ of the robot end effector with respect to the fixed frame in such a way that the estimated image position $\widehat{X}_{p / a}$ follows a given scanning trajectory $X_{d}(t)$. Usually, in visual servoing, when the bandwidth is not an issue (which is the case here since the displacements are very slow), a simple proportional controller is used to obtain an exponential convergence of the servoed error towards zero. Additionally, a feedforward term is used when the desired image velocity is not null. This approach is appropriate in conventional situations when any motion of the imaging device produces a motion in the image. However, here, tissue deformations play the role of an external disturbance. In order to reject it, since again bandwidth is not a crucial issue, a simple integral term is used, assuming that the disturbance is quasi-static.

The resulting control law writes:

$$
\begin{array}{r}
V_{r / 0}=\underbrace{\frac{\mathrm{d} X_{d}}{\mathrm{~d} t}}_{\text {Feedforward }}+\underbrace{K_{P}\left(X_{d}(t)-\widehat{X}_{p / a}(t)\right)}_{\text {Proportional }}+ \\
\underbrace{K_{I} \int_{0}^{t}\left(X_{d}(t)-\widehat{X}_{p / a}(\tau)\right) d \tau}_{\text {Integral }}
\end{array}
$$

where $K_{P}$ and $K_{I}$ are the proportional and the integral gain, respectively. The parameters are tuned in a standard way [19], resulting in a proportional gain of $3 s^{-1}$ and an integral gain of $0.15 \mathrm{~s}^{-2}$. This provides a response time of less than $1 \mathrm{~s}$ with proper damping.

The control scheme is presented in Figure 7.

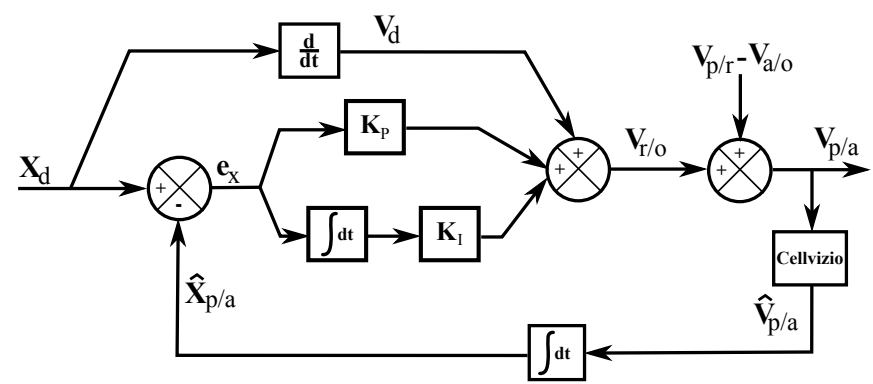

Fig. 7. The proposed control scheme

\section{B. Results}

For comparison with the experiments presented in Sec. III and Sec. IV, the 8-shape experiment is performed on a piece of beef liver using the vision based control algorithm. Namely, the desired trajectory $X_{d}(t)$ for the visual servoing loop is now the 8-shape trajectory that was previously sent directly to the robot.

It can be seen in Fig. 8 that the image trajectory is following the reference trajectory with very high precision: the final error between the first and the last image is $15 \mu \mathrm{m}$ and the mean tracking position error is $30 \mu \mathrm{m}$. This improvement is due to the fact that the tissue and the probe
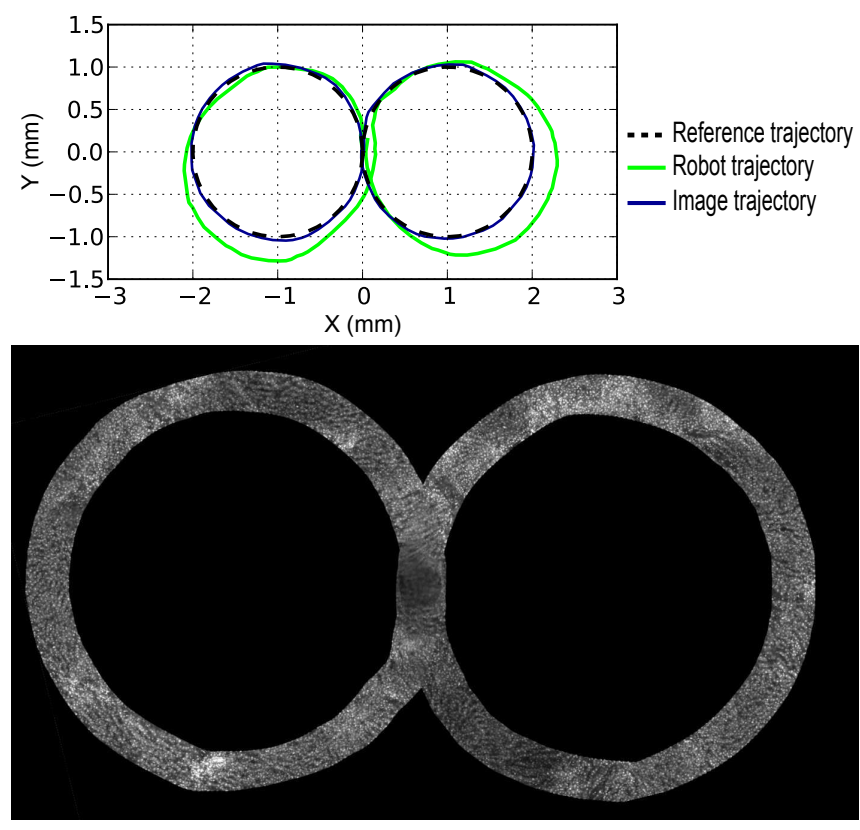

Fig. 8. 8 shape trajectory on a piece of beef liver using the visual servoing algorithm. Up: robot and image XY trajectories. Down: computed mosaic

holder deformations are rejected by the control algorithm thanks to the integral term. Notice that the reported $15 \mu \mathrm{m}$ error is not the servoed error, which is null due to the integral term. It reports the measured displacement between the initial and the final images, which is not null due to a slight drift in the image-based position estimation process that arises from the time integration of the measured velocity. In other words, the servoed error is null but the position measurement is wrong by $15 \mu \mathrm{m}$.

In Fig. 8 it is also interesting to observe the robot trajectory (green line) which sums up the image trajectory and the compensation for deformations provided by the integral term in the compensator. Clearly, it seems that this deformation, that may be as large as a few hundreds $\mu \mathrm{m}$, is hardly predictable for complex trajectory shapes. This justifies, $a$ posteriori, the use of a model-free, integral based approach for the disturbance rejection.

In order to assess the influence of abrupt changes in the direction of the reference trajectory, a $3 \mathrm{~mm}$ large square scan is made on a piece of beef liver using the position control algorithm. Figure 9 shows a sample mosaic computed from the images along with the desired trajectory. The straight lines are correctly followed, and the final error is less than $40 \mu \mathrm{m}$. One can also notice the transient behavior at direction changes.

Finally, since the aim of this study is to provide a solution for making shape-controlled optical biopsies with confocal imaging, a surface scan of a complete area is performed on a piece of beef liver. The trajectory is a $1 \times 1 \mathrm{~mm}$ raster scan, like in Fig. 6. Figure 10 presents the result. The mosaic does not exhibit holes and has the expected square shape. Figure 11 presents the results of the same experiment on a muscle tissue, namely a piece of chicken breast. The 


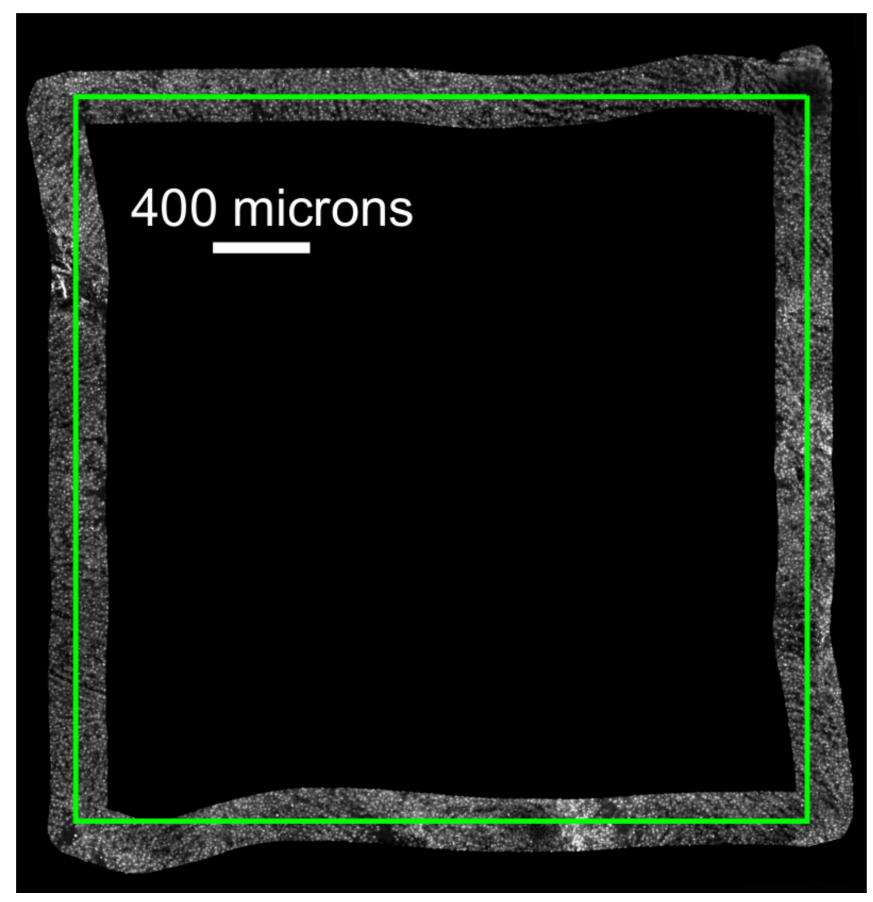

Fig. 9. Mosaic obtained by following a $3 \mathrm{~mm}$ large square scan on beef liver using the position control algorithm. The green line is the desired trajectory

mosaic quality is here also satisfactory. Since the mechanical properties of muscle tissue are very different from those of liver tissue [20], this proves that our control algorithm is robust to variations in mechanical tissue properties, as the integral term rejects low frequency disturbances without requiring any model.

\section{CONCLUSION}

In this paper, we focused on controlled navigation of a rigid confocal probe over the surface of a deformable tissue. We proposed and validated the use of an image-based speed measurement as a feedback signal for controlling the probe trajectory. To our knowledge, a first contribution is the yet unpublished extension of marker-free visual servoing to a new form of medical imaging.

A particular problem that has to be taken into account is the tissue deformation phenomenon, which is evidenced in the paper and acts as a disturbance to be rejected by the controller. A simple PI control loop with a feedforward action appeared to be sufficient for rejecting unmodelled tissue deformation disturbances under varying conditions. As a result, the position error on soft tissue is reduced to a few microns when following the trajectory, with a null steady state error. Surface scans have been realized on the surface of different animal ex-vivo tissues with satisfactory results using a precision industrial robot and the developed control algorithm.

Further works are directed towards studying the impact of the trajectory (angles, accelerations) on the tissue deformations, in order to design scan trajectories that minimize

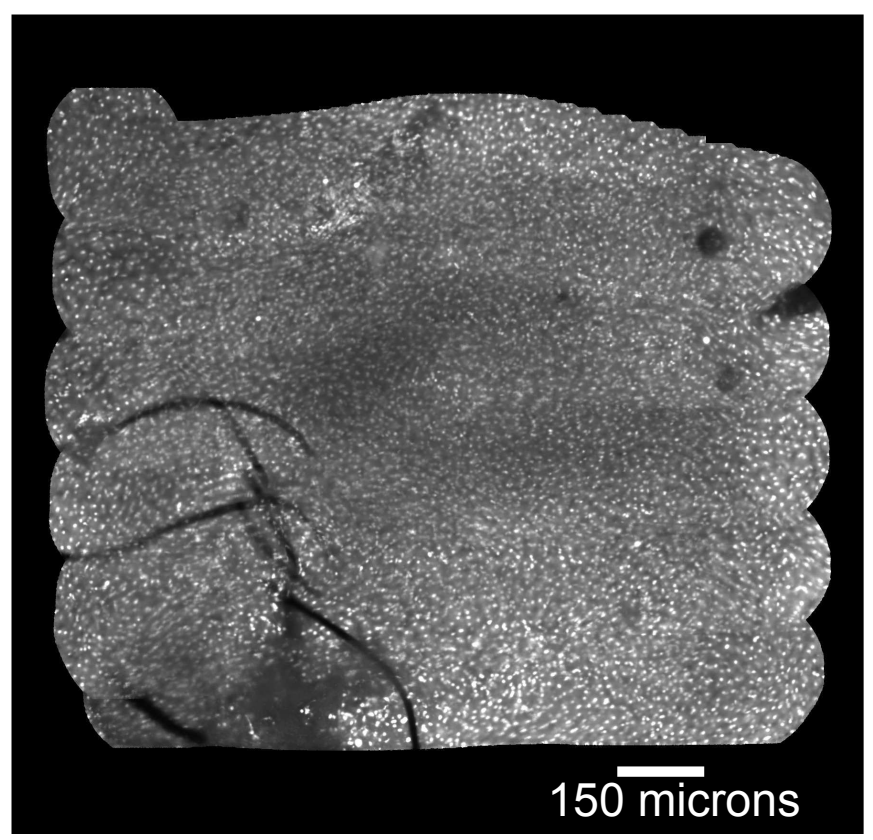

Fig. 10. Mosaic obtained by following a raster scan on beef liver with control

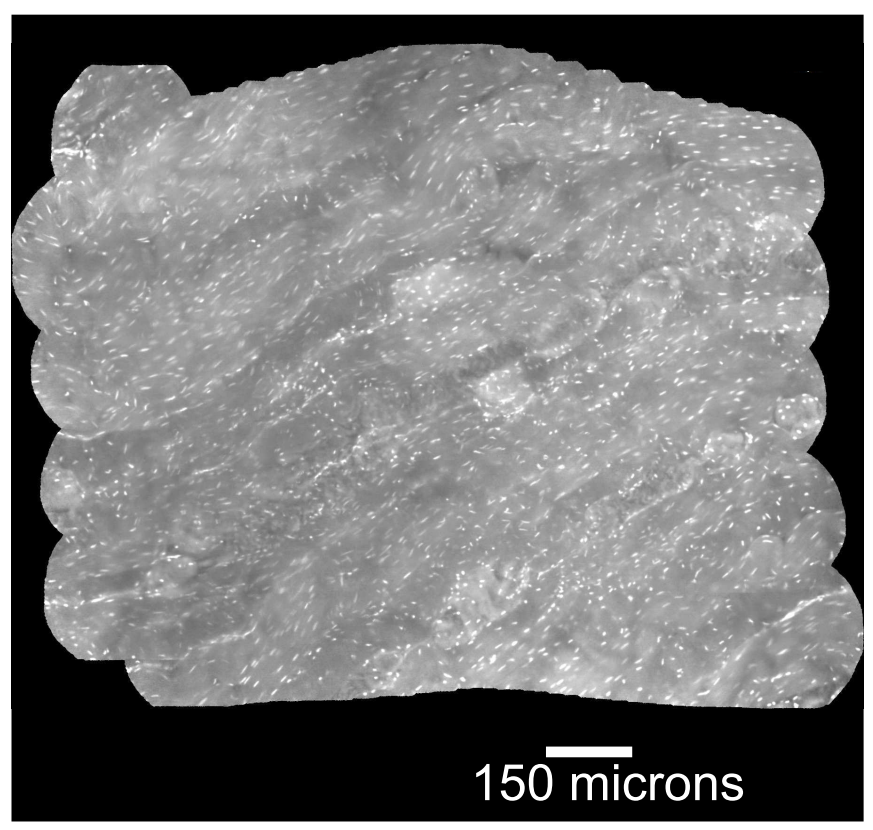

Fig. 11. Mosaic obtained by following a raster scan on chicken breast with control

disturbances. Adding a force control loop for controlling the probe pressure on the tissue is also considered.

Most importantly, in vivo exploration of tissues is targeted in this project. In this paper, an industrial precision robot was used to demonstrate the effectiveness of our visual servoing approach for the compensation of tissue deformations during a scan. Ex vivo experiments were carried out with this robot. However, this experimental platform is not appropriate for in vivo experiments as a minimally invasive access is required. To this aim, the control algorithm is to be implemented on a 
dedicated laparoscopic instrument that has the capability of stably and smoothly sweeping the surface of moving organs. A candidate for this further development is the hydraulically actuated laparoscopic instrument that we recently presented in [11], where the stabilization and sweeping capacities have been demonstrated in open loop during in vivo experiments. It is anticipated that modifications on both the image processing algorithm and the controller may be necessary since the quality of the robotic probe motion control may be degraded as compared to the motion produced by the high precision industrial robot used in the present paper.

\section{ACKNOLEDGEMENTS}

This work was supported by OSEO (Maisons-Alfort, France) under ISI Project PERSEE (number I0911038W), which partners are Mauna Kea Technologies (project leader, Paris, France), Endocontrol (Grenoble, France), Institut $\mathrm{Mu}$ tualiste Montsouris (Paris, France), Institut Gustave Roussy (Villejuif, France) and ISIR-UPMC (Paris, France).

The project also largely benefits from the interaction with three anthropologists, Marie-Christine Pouchelle, Caroline Moricot and Marina Maestrutti, who are warmly thanked (support from CNRS, PE/PS program of INSHS and from INCA - the national institute of cancer).

\section{REFERENCES}

[1] D. Huang, E. Swanson, C. Lin, J. Schuman, W. Stinson, W. Chang, M. Hee, T. Flotte, K. Gregory, C. Puliafito et al., "Optical coherence tomography," Science, vol. 254, no. 5035, p. 1178, 1991.

[2] M. Wallace, P. Fockens et al., "Probe-based confocal laser endomicroscopy." Gastroenterology, vol. 136, no. 5, p. 1509, 2009.

[3] Y. Sabharwal, A. Rouse, L. Donaldson, M. Hopkins, and A. Gmitro, "Slit-scanning confocal microendoscope for high-resolution in vivo imaging," Applied optics, vol. 38, no. 34, pp. 7133-7144, 1999.

[4] W. Piyawattanametha, H. Ra, M. Mandella, K. Loewke, T. Wang, G. Kino, O. Solgaard, and C. Contag, "3-d near-infrared fluorescence imaging using an mems-based miniature dual-axis confocal microscope," Selected Topics in Quantum Electronics, IEEE Journal of, vol. 15 , no. 5, pp. 1344-1350, 2009.

[5] R. Kiesslich, L. Gossner, M. Goetz, A. Dahlmann, M. Vieth, M. Stolte, A. Hoffman, M. Jung, B. Nafe, P. Galle et al., "In vivo histology of barrett's esophagus and associated neoplasia by confocal laser endomicroscopy," Clinical Gastroenterology and Hepatology, vol. 4, no. 8, pp. 979-987, 2006.
[6] A. Polglase, "A fluorescence confocal endomicroscope for in vivo microscopy of the upper- and the lower-GI tract," Gastrointestinal Endoscopy, vol. 62, no. 5, 2005.

[7] A. Tanbakuchi, A. Rouse, J. Udovich, K. Hatch, and A. Gmitro, "Clinical confocal microlaparoscope for real-time in vivo optical biopsies," Journal of biomedical optics, vol. 14, p. 044030, 2009.

[8] T. Vercauteren, A. Perchant, G. Malandain, X. Pennec, and N. Ayache, "Robust mosaicing with correction of motion distortions and tissue deformations for in vivo fibered microscopy," Medical Image Analysis, vol. 10, no. 5, pp. 673-692, Oct. 2006.

[9] K. E. Loewke, D. B. Camarillo, W. Piyawattanametha, M. J. Mandella, C. H. Contag, S. Thrun, and J. K. Salisbury, "In vivo micro-image mosaicing," IEEE Trans. Biomed. Eng., vol. 58, no. 1, pp. 159-171, Jan. 2011

[10] W. Latt, R. Newton, M. Visentini-Scarzanella, C. Payne, D. Noonan, J. Shang, and G. Yang, "A hand-held instrument to maintain steady tissue contact during probe-based confocal laser endomicroscopy," Biomedical Engineering, IEEE Transactions on, vol. 58, no. 9, pp. 2694-2703, 2011.

[11] B. Rosa, B. Herman, J. Szewczyk, B. Gayet, and G. Morel, "Laparoscopic optical biopsies: in vivo robotized mosaicing with probe-based confocal endomicroscopy," in Intelligent Robots and Systems (IROS), 2011 IEEE/RSJ International Conference on. IEEE, 2011, pp. 13391345.

[12] M. Baumhauer, M. Feuerstein, H. Meinzer, and J. Rassweiler, "Navigation in endoscopic soft tissue surgery: perspectives and limitations," Journal of Endourology, vol. 22, no. 4, pp. 751-766, 2008.

[13] X. Liu, S. Xu, H. Zhang, and L. Hu, "A new hybrid soft tissue model for visio-haptic simulation," Instrumentation and Measurement, IEEE Transactions on, no. 99, pp. 1-12, 2011.

[14] B. Tay, J. Kim, and M. Srinivasan, "In vivo mechanical behavior of intra-abdominal organs," Biomedical Engineering, IEEE Transactions on, vol. 53, no. 11, pp. 2129-2138, 2006.

[15] T. Vercauteren, A. Meining, F. Lacombe, A. Perchant, J. Conchello, C. Cogswell, T. Wilson, and T. Brown, "Real time autonomous video image registration for endomicroscopy: fighting the compromises," in SPIE BIOS - Three-Dimensional and Multidimensional Microscopy: Image Acquisition and Processing XV, vol. 6861, 2008, p. 68610C.

[16] J. P. Lewis, "Fast template matching," in Proceedings of the International Conference on Vision Interface (VI'95), 1995, pp. 120-123.

[17] M. Tada, M. Mochimaru, and T. Kanade, "How does a fingertip slip? - contact mechanics of a fingertip under tangential loading -," in Proc. of EuroHaptics 2006, jul 2006, pp. 415-420.

[18] A. Terekhov and V. Hayward, "Minimal adhesion surface area in tangentially loaded digital contacts," Journal of biomechanics, vol. 44, no. 13 , pp. 2508-2510, 2011.

[19] A. O'Dwyer, Handbook of PI and PID controller tuning rules. Imperial College Press, 2009.

[20] V. Egorov, S. Tsyuryupa, S. Kanilo, M. Kogit, and A. Sarvazyan, "Soft tissue elastometer," Med Eng Phys, vol. 30, no. 2, pp. 206-212, Mar 2008 . 